\title{
Towards Self-Parameterized Active Contours for Medical Image Segmentation with Emphasis on Abdomen
}

\author{
Eleftheria A. Mylona, Michalis A. Savelonas, and Dimitris Maroulis
}

\begin{abstract}
Medical doctors are typically required to segment medical images by means of computational tools, which suffer from parameters that are empirically selected through a cumbersome and time-consuming process. This chapter presents a framework for automated parameterization of region-based active contour regularization and data fidelity terms, which aims to relieve medical doctors from this process, as well as to enhance objectivity and reproducibility. Leaned on an observed isomorphism between the eigenvalues of structure tensors and active contour parameters, the presented framework automatically adjusts active contour parameters so as to reflect the orientation coherence in edge regions by means of the "orientation entropy." To this end, the active contour is repelled from randomly oriented edge regions and is navigated towards structured ones, accelerating contour convergence. Experiments are conducted on abdominal imaging domains, which include colon and lung images. The experimental evaluation demonstrates that the presented framework is capable of speeding up contour convergence, whereas it achieves high-quality segmentation results, albeit in an unsupervised fashion.
\end{abstract}

\section{Introduction}

Medical image segmentation is an essential instrument in computer-aided diagnosis, being potentially crucial for localization of pathologies, study of anatomical structures, computer-integrated surgery, and treatment planning. In particular, abdominal image segmentation allows medical doctors (MDs) to investigate abdominal organs, as visualized by noninvasive imaging modalities. As part of their clinical diagnosis, MDs are typically required to examine and

E.A. Mylona • M.A. Savelonas • D. Maroulis $(\bowtie)$

Department of Informatics and Telecommunications, University of Athens,

Panepistimiopolis, Ilissia 15703, Athens, Greece

e-mail: emylona@di.uoa.gr; msavel@di.uoa.gr; dmaroulis@di.uoa.gr 
interpret abdominal images obtained by CT scans, in order to extract vital information on abdominal organs, which is associated with their anatomy and pathology. Although such images may contain detailed information, they are often plagued by noise, artifacts, as well as heterogeneity, which yield to inhomogeneous background.

Medical image segmentation has to be a robust and reproducible process without human intervention, so as to substantially support diagnosis and clinical evaluation. However, most segmentation methods are highly parametric, and human intervention is often inevitable. In this regard, automatic medical image segmentation techniques are in demand, so as to ease MDs' workload and bolster the objectivity of the segmentation results.

Region-based active contour models are widely applied for medical image segmentation due to their inherent noise-filtering mechanism and their topological adaptability. Moreover, they are robust to weak edges and intensity inhomogeneity [1-4]. Researchers have developed various region-based active contour variations for abdominal image segmentation. Dhalila et al. [5] propose a semiautomatic active contour variation for the segmentation of the abdominal region of the human body. In the first phase, user intervention is a prerequisite for manual segmentation of a certain number of slices, whereas in the second phase, segmentation is automatic. Jiang et al. [6] propose an approach based on active contour for segmentation of the liver region in abdominal CT images. The active contour model is combined with threshold and morphology-based techniques in order to extract the initial contour and segment the liver slice by slice. Plajer et al. [7] present an active contour algorithm for lung tumor segmentation in 3D-CT image data. The algorithm is based on a mixed internal-external force as well as on a cluster function.

The development of such powerful computational tools contributes to the early diagnosis of the pathology in abdominal organs. However, the vast majority of these tools are dominated by parameters, and although these parameters have a major impact on the segmentation quality, they are empirically determined through the tedious and time-consuming process of trial and error. Parameters are often selected on the basis of a limited amount of experimental results and the visual impression of the domain user, whereas they may be valid for a specific dataset. To this end, the objectivity and reproducibility of the segmentation results are highly questioned. Furthermore, empirical parameterization presumes certain technical knowledge by the end user with respect to the algorithm's intrinsic mechanisms. Nevertheless, this is not the case in the context of medical imaging where the end user is usually a MD.

\section{Previous Work}

Several region-based active contour variations have been developed in order to tackle with empirical parameterization. Ma and Yu [8] attempt to balance regionbased forces by means of mathematical morphology without separately adjusting 
each individual parameter. McIntosh and Hamarneh [9] adapt regularization weights across a set of images. Although one weight value may be optimal for some regions in an image, it may not be optimal for all regions. Erdem and Tari [10] and Kokkinos et al. [11] focus on edge consistency and texture cues by utilizing data-driven local cues. However, certain technical knowledge by the domain user is still required. Pluemptiwiriyaweg et al. [12] and Tsai et al. [13] dynamically update active contour parameters during contour evolution. Nonetheless, possible erroneous behavior of the contour in the early stages of evolution, with effects on convergence, has not been considered. Furthermore, parameters are not spatially adaptive, failing to capture local image content. Keuper et al. [14] and Liu et al. [15] propose a method for dynamic adjustment of active contour parameters, applicable on the detection of cell nuclei and lip boundaries, respectively. Both methods require a priori knowledge considering the shape of the target region. Iakovidis et al. [16] and Hsu et al. [17] introduce a framework for optimization of active contour parameters based on genetic algorithms. However, these heuristic approaches converge slowly in locally optimal solutions. Allili et al. [18] present an approach for estimating hyper-parameters capable of balancing the contribution of boundary and region-based terms. In their approach, empirical parameter tuning is still involved. Yushkevich et al. [19] develop an application for level-set segmentation of anatomical structures. Although their GUI is friendly to non-expert users, parameter settings are still empirically determined. Dong et al. [20] present an algorithm to capture brain aneurysms from the vascular tree, by varying the regularization term based on the surface curvature of a pre-segmented vessel. However, the regularization weight does not rely on image content. On the contrary, it depends on the shape of the target region, thus limiting the applicability of the method on different target shapes.

This chapter presents a framework for automated parameterization of region-based active contours, which is applicable on medical image segmentation. The presented framework is inspired by the observation of an isomorphism between the eigenvalues of structure tensors and the active contour regularization and data fidelity parameters. The latter are capable of describing the orientation coherence of edge regions similarly to the former by means of the measure called orientation entropy (OE). This measure obtains low values in structured regions, which contain edges with low orientation variability, and high values in unstructured regions, which contain edges of multiple orientations. Accordingly, OE is capable to adjust forces driving the contour away from unstructured edge regions and guide it towards more structured ones, which are naturally associated with the boundaries of medical objects. Hence, iterations dedicated to false local minima are bypassed, speeding up contour convergence.

The presented framework aims to:

(a) Relieve MDs from the cumbersome and time-consuming process of empirical parameterization

(b) Cope well with the large variability of the shape of target regions in abdominal images 
(c) Remain insensitive to noise, artifacts, and heterogeneity

(d) Provide objectivity and reproducibility

\section{Parameter-Adjustment Framework}

The presented parameter-adjustment framework exploits the attractive properties of structure tensor eigenvalues.

\section{Structure Tensors}

Structure tensors [21] have been extensively utilized in image analysis for various tasks such as anisotropic filtering [22] and motion detection [23].

In Weickert's diffusion model [24], the structure tensor $D$ is a symmetric, semi-positive $2 \times 2$ matrix (also called "second-moment matrix"), capable of describing the orientation coherence of an edge region and is defined as

$$
D=\left(\begin{array}{ll}
v_{1} & v_{2}
\end{array}\right)\left(\begin{array}{cc}
\lambda_{1} & 0 \\
0 & \lambda_{2}
\end{array}\right)\left(\begin{array}{ll}
v_{1} & v_{2}
\end{array}\right)^{\mathrm{T}}=\left(\begin{array}{ll}
d x & d y
\end{array}\right)\left(\begin{array}{cc}
I_{x x} & I_{x y} \\
I_{y x} & I_{y y}
\end{array}\right)\left(\begin{array}{ll}
d x & d y
\end{array}\right)^{\mathrm{T}}
$$

where $I$ is the input image, $v_{1}, v_{2}$ are orthonormal eigenvectors, and $\lambda_{1}, \lambda_{2}$ are the corresponding eigenvalues given by

$$
\lambda_{1,2}=\frac{1}{2}\left(I_{x x}+I_{y y} \pm \sqrt{\left(I_{x x}-I_{y y}\right)^{2}+4 I_{x y}^{2}}\right)
$$

where the + sign belongs to $\lambda_{1}$. The eigenvectors and eigenvalues of the structure tensor reflect the local orientation of edge regions. The eigenvectors form the orthogonal basis so that the variance of the projection on one of the tensor's axes is maximal and the projection on one of the remaining axes is minimal [25]. The eigenvalues describe the orientation coherence along the corresponding eigenvectors. It is worth to be noted that $\lambda_{1}$ is the principal eigenvalue and is longitudinal with respect to the principal axis of the tensor ellipsoid, whereas $\lambda_{2}$ is the minor eigenvalue and is vertical with respect to the same principal axis. Figure 1 depicts an elliptical representation of a 2D structure tensor.

Providing that an image region contains either edges of approximately the same orientation, or edges of multiple orientations, it can be identified by means of a structure tensor as a structured or unstructured edge region, respectively. The boundaries of medical objects are naturally associated with structured edge regions, whereas unstructured edge regions are associated with noise, artifacts, and/or background clutter. In this light, structure tensors are capable of providing maps of target and nontarget edge regions in the context of a medical imaging application. 
Fig. 1 Elliptical representation of a $2 \mathrm{D}$ structure tensor

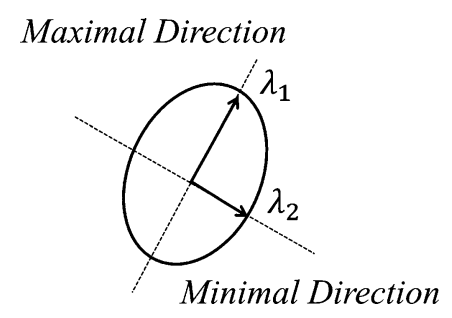

Maximal Direction

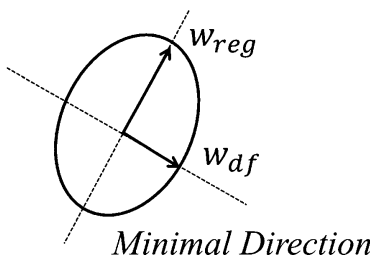

Minimal Direction
Fig. 2 Elliptical representation of active contour

\section{Region-Based Active Contours}

The energy functional of the region-based active contours that is minimized can be written as follows:

$$
E_{\text {total }}=w_{\text {reg }} \cdot E_{\mathrm{reg}}+w_{\mathrm{df}} \cdot E_{\mathrm{df}}
$$

where $E_{\text {reg }}$ and $E_{\mathrm{df}}$ are the regularization and data fidelity energy terms, respectively, whereas $w_{\text {reg }}$ and $w_{\text {df }}$ are the corresponding weighting parameters. Energy terms are scalar functions, which most often discard any information associated with the orientation coherence of edge regions. However, forces guiding contour evolution are vectors which are affected by the orientation coherence of edges.

Regularization forces are tangent with respect to the principal axis of the contour, whereas data fidelity forces are vertical, attracting the contour towards target edges. Providing that the contour is initialized as an ellipsoid, the regularization weight $w_{\text {reg }}$ is longitudinal with respect to the principal axis of the contour, whereas the data fidelity weight $w_{\mathrm{df}}$ is vertical with respect to the same principal axis. Figure 2 depicts an elliptical representation of an active contour.

It can be noted that the regularization weight $w_{\text {reg }}$ corresponds to the same direction as the principal eigenvalue $\lambda_{1}$, whereas the data fidelity weight $w_{\mathrm{df}}$ corresponds to the same direction as the minor eigenvalue $\lambda_{2}$. This isomorphism associates the regularization and data fidelity parameters with the eigenvalues of the structure tensor. 


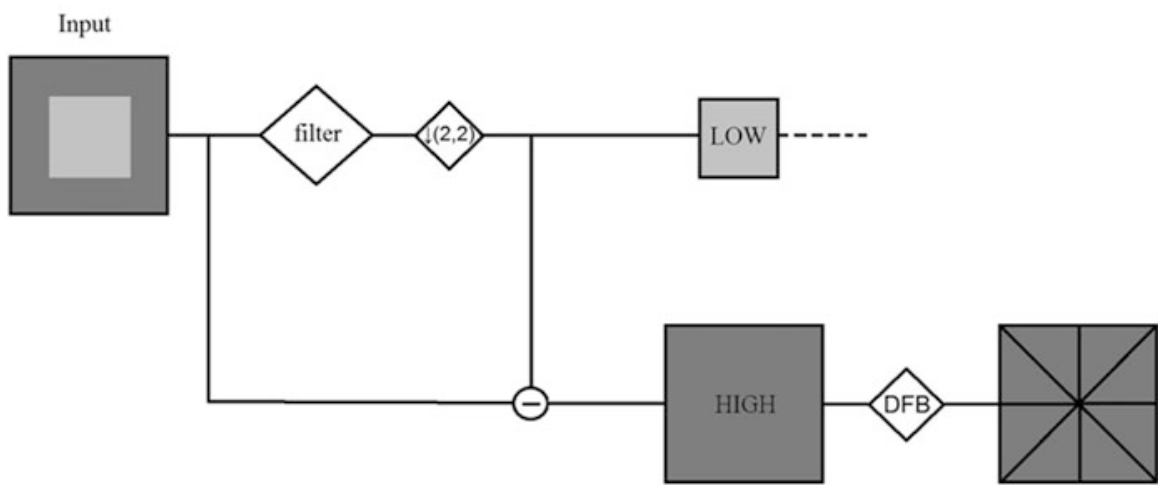

Fig. 3 CTr iterated filter bank. LP provides a downsampled low-pass version and a band-pass version of the image. Consequently, a DFB is applied to each band-pass image

\section{Orientation Estimation}

Inspired by the aforementioned observation, regularization and data fidelity parameters of region-based active contours are automated in order to reflect the orientation coherence of edge regions, in a similar fashion to Weickert's diffusion model [24]. The orientation coherence is estimated by means of the orientation entropy (OE). The latter is calculated on directional subbands in each scale of the contourlet transform (CTr) [26], which, apart from intensity, also represents textural information. This approach provides an inherent filtering mechanism, capable of filtering out randomly oriented edges associated with noise, artifacts, and/or background clutter. Moreover, CTr is directly implemented in the discrete domain, as opposed to similar transforms, such as curvelets [27].

\section{The Contourlet Transform}

$\mathrm{CTr}$ is an anisotropic directional image representation scheme, which effectively quantifies diffusion over contour segments with varying elongated shapes and directions. Aiming at a sparse image representation, it employs a double iterated filter bank, which captures point discontinuities by means of the Laplacian pyramid (LP) and obtains linear structures by linking these discontinuities with a directional filter bank (DFB). The final result is an image expansion that uses basic contour segments. Figure 3 illustrates a CTr iterated filter bank.

The downsampled low-pass and band-pass versions of the image contain lower and higher frequencies, respectively. It is evident that the band-pass image contains detailed information of point discontinuities which are associated with target edges. Furthermore, DFB is implemented by an l-level binary tree which leads to $2^{l}$ 


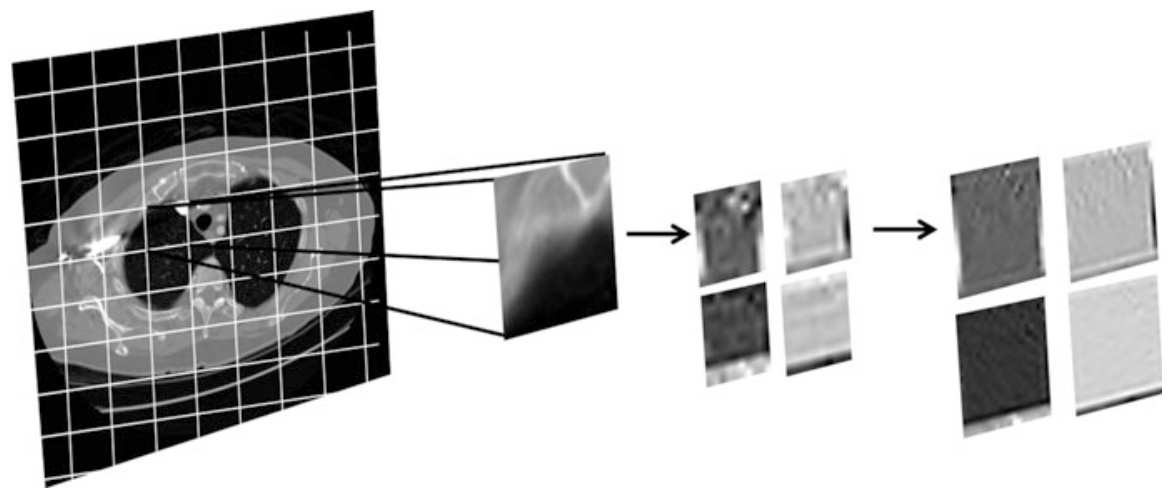

Fig. 4 CTr filter bank on a sample grid of a lung CT scan decomposed to two levels of LP and four band-pass directional subbands

subbands. In the first stage, a two-channel quincunx filter bank [28] with fan filters divides the 2D spectrum into vertical and horizontal directions.

In the second stage, a shearing operator reorders the samples. As a result, different directional frequencies are captured at each decomposition level. The number of iterations depends mainly on the size of the input image. The total number of directional subbands $K_{\text {total }}$ is calculated as

$$
K_{\text {total }}=\sum_{j=1}^{J} K_{j}
$$

where $K_{j}$ is a subband DFB applied at the $j$ th level $(j=1,2, \ldots, J)$.

Figure 4 depicts the CTr filter bank applied on a sample grid of a lung CT scan, decomposed to the finest and second finest scales which are partitioned into four directional subbands. Each $q \times q$ image grid is fed into the CTr filter bank through an iterative procedure. This grid must be appropriately selected in order to preserve the orientation of the main structures of the target region. The band-pass directional subbands represent the local image structure. It should be mentioned that the presented framework is not confined in using CTr and could also embed alternative multi-scale or multi-directional approaches for image representation.

In the context of the presented framework, OE is calculated for each subband image $I_{j k}$ as follows:

$$
\begin{gathered}
\mathrm{OE}_{j k}=-\sum_{n=1}^{N_{j k}} \sum_{m=1}^{M_{j k}} p_{j k}(m, n) \cdot \log p_{j k}(m, n) \\
p_{j k}(m, n)=\frac{\left|I_{j k}(m, n)\right|^{2}}{\sqrt{\sum_{n=1}^{N_{j k}} \sum_{m=1}^{M_{j k}}\left[I_{j k}(m, n)\right]^{2}}}
\end{gathered}
$$




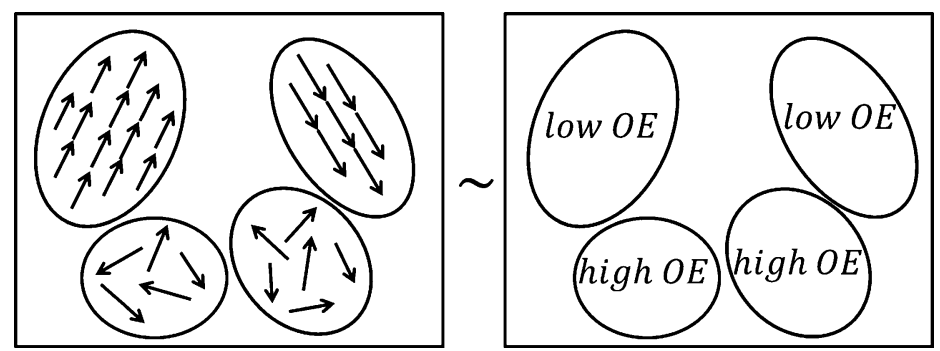

Fig. 5 Schematic representation of (a) elliptical structure tensors and (b) OE behavior on each structure tensor

where $\mathrm{OE}_{j k}$ is the $\mathrm{OE}$ of the subband image $I_{j k}$ in the $k$ th direction and the $j$ th level of decomposition, $M_{j k}$ is the row size, and $N_{j k}$ is the column size of the subband image. OE obtains high and low values in cases of unstructured, nontarget and structured, target edge regions, respectively. Figure 5a depicts a schematic representation of several elliptical structure tensors consisting of single and multiple orientations, whereas Fig. 5b depicts the OE behavior on each structure tensor of Fig. 5a.

\section{Automated Parameter Adjustment}

Regularization and data fidelity parameters are automatically adjusted according to the following equations:

$$
w_{\text {reg }}^{\text {auto }} \propto\left(1 / w_{\mathrm{df}}\right) \times N \times M, \quad w_{\mathrm{df}}^{\text {auto }}=\arg _{I_{j k}} \max \left(\mathrm{OE}_{j k}\left(I_{j k}\right)\right)
$$

The core idea is to guide the active contour towards structured, target edge regions in the early stages of evolution by appropriately amplifying data fidelity forces in randomly oriented, high-entropy regions. As a result the contour will be repelled and iterations dedicated to erroneous local minima will be bypassed, speeding up contour convergence towards target edges. Equation (7) is an interpretation of orientation entropy values adaptive to the orientation of data fidelity forces. Apart from separately adjusting each parameter, the presented framework also achieves a balanced trade-off between regularization and data fidelity parameters. It should also be noticed that the automated parameterization is spatially adaptive, so as to reflect local variations over the image.

Figure 6 illustrates the improvement in contour evolution achieved by the presented framework as compared to the typical contour evolution obtained by empirical parameterization. Figure 6a depicts a synthetic image containing a target 

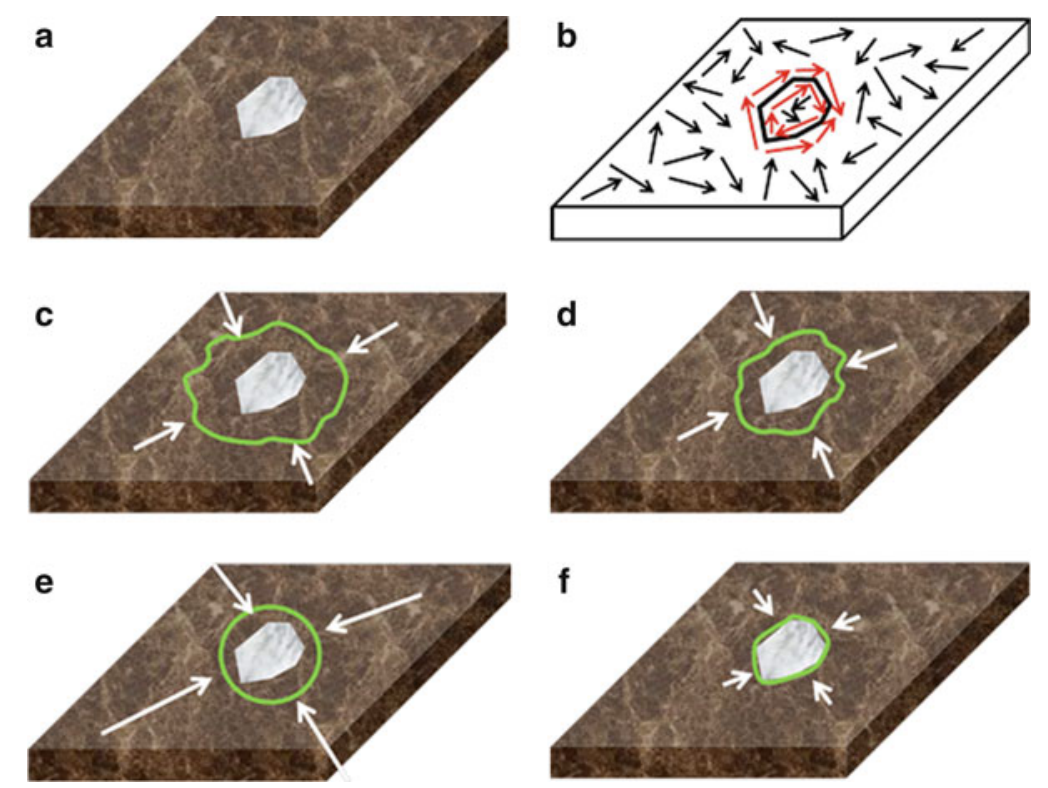

Fig. 6 Contour evolution of the presented framework vs. empirical parameterization, (a) synthetic image, (b) sketch of orientation variability, $(\mathbf{c}, \mathbf{d})$ evolution of empirical parameterization, and $(\mathbf{e}, \mathbf{f})$ evolution of the presented framework

region over an inhomogeneous background, resembling a typical medical image case, whereas Fig. $6 \mathrm{~b}$ depicts a sketch of orientation variability of the synthetic image. Red arrows correspond to structured, target edge regions, whereas black ones indicate unstructured, nontarget ones.

In the case of empirical parameterization, the contour will be trapped in false local minima associated with background clutter in the early stages of evolution (Fig. 6c). Since region-based forces (short white arrows) are uniformly weighted irrespectively of $\mathrm{OE}$, the contour will be kept away from target edge regions for more iterations (Fig. 6d).

In the case of automated parameterization, OE is considered. For as long as the contour lies in unstructured edge regions associated with background clutter, OE obtains high values and the data fidelity parameter is increased. Thus, region-based forces (long white arrows) are appropriately amplified, repelling the contour away from such regions and navigating it towards more structured ones (Fig. 6e). Once the contour approximates the vicinity of structured edge regions, OE obtains low values and the data fidelity parameter is decreased. Hence, regionbased forces (short white arrows) are appropriately reduced in order to facilitate convergence (Fig. 6f). To this end, contour convergence is achieved in less iterations. 


\section{Results}

The presented framework is embedded into the Chan-Vese model [29] by replacing the optimal fixed parameters with the automatically adjusted parameters, in order to evaluate the segmentation performance of the automated vs. the empirically finetuned version. The Chan-Vese model determines the level set evolution by solving the following equation:

$$
\begin{aligned}
\frac{\partial \phi}{\partial t}= & w_{\text {reg }}^{\text {fixed }} \cdot \delta(\phi(x, y)) \cdot \operatorname{div}\left(\frac{\nabla \phi}{|\nabla \phi|}\right)-w_{\mathrm{df}}^{\mathrm{fixed}}\left(I(x, y)-c_{1}\right)^{2} \\
& +w_{\mathrm{df}}^{\mathrm{fixed}}\left(I(x, y)-c_{2}\right)^{2}
\end{aligned}
$$

where $\phi$ is the level set function, $I$ the observed image, $c_{1}, c_{2}$ the average intensities inside and outside of the contour, respectively, $w_{\text {reg }}^{\text {fixed }}$ the fixed regularization parameter, and $w_{\mathrm{df}}^{\text {fixed }}$ the fixed data fidelity parameter. For the empirical case, the optimal parameters are set according to the original paper [29]. For the presented framework, the regularization and data fidelity parameters are automatically calculated according to (7).

Experiments are conducted on three datasets consisting of abdominal imaging modalities such as colon and lung images. Additional experiments are conducted on one dataset containing mammographic images in order to evaluate the presented framework on a different imaging modality comprising abnormalities of various sizes and shapes. All imaging modalities were investigated by MD experts who provided ground truth images.

The first dataset consists of 32 endoscopy frame images containing polyps provided by the Gastroenterology Section, Department of Pathophysiology, Medical School, University of Athens, Greece, and partially by the Section for Minimal Invasive Surgery, University of Tübingen, Germany. The endoscopic data was acquired from sixty-six different patients with an Olympus CF-100 HL endoscope. All frame images consist of small-size adenomatous polyps which are not easily detectable and are more likely to become malignant.

The second dataset consists of 30 axial CT scans of the lung parenchyma obtained by the lung image dataset consortium image collection (LIDC-IDRI) [30]. The aim of segmentation is to separate the lung parenchyma from the surrounding anatomy, which is typically impeded by airways or other "airwaylike" structures in the right and left lung. The segmentation result is used for the computation of emphysema measures.

The third dataset consists of $26 \mathrm{CT}$ scans of the thorax obtained by the NSCLC Radiogenomics collection [30]. The segmentation result is used for the evaluation of the condition of the lungs and for further physiological measurements.

The fourth dataset consists of 50 mammographic images containing abnormalities randomly obtained by the Mini-MIAS dataset [31]. The background tissue is characterized as (a) fatty, (b) fatty glandular, and (c) dense glandular, 


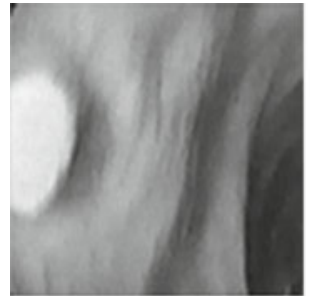

(a)

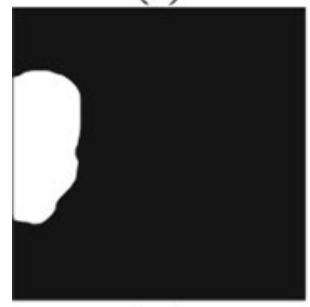

$\left(a_{1}\right)$

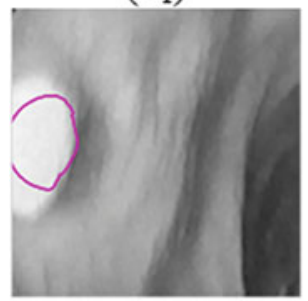

$\left(a_{2}\right)$

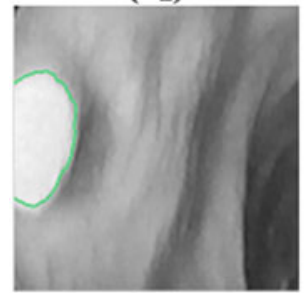

$\left(a_{3}\right)$

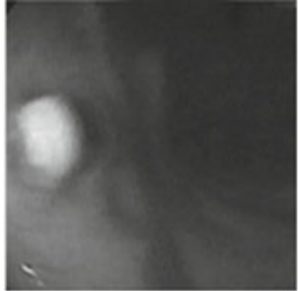

(b)

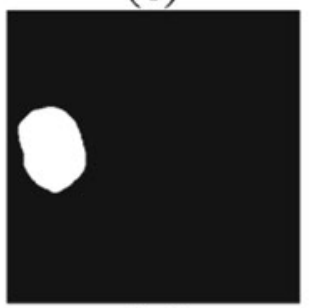

$\left(b_{1}\right)$

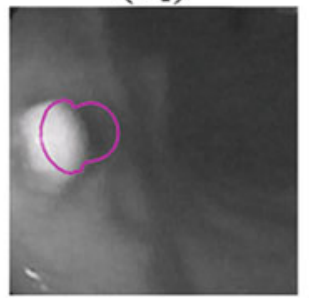

$\left(b_{2}\right)$

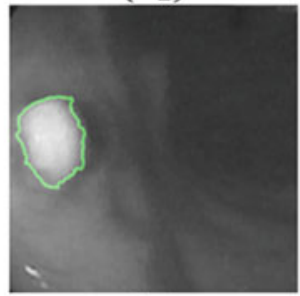

$\left(b_{3}\right)$

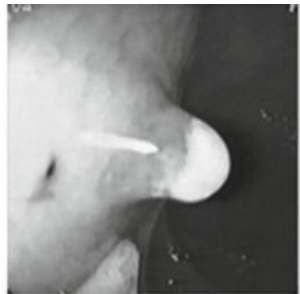

(c)

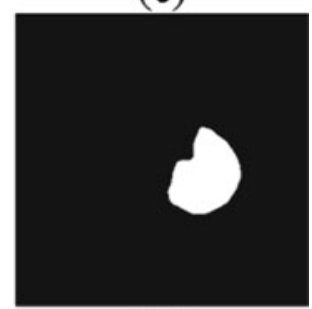

$\left(c_{1}\right)$

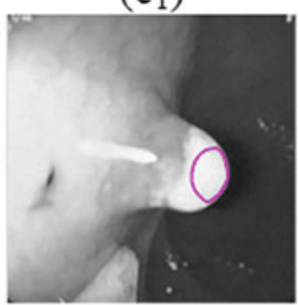

(c)

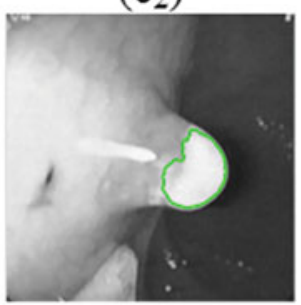

$\left(c_{3}\right)$

Fig. 7 (a-c) Endoscopy images containing polyps, $\left(\mathbf{a}_{1}-\mathbf{c}_{1}\right)$ ground truth images, $\left(\mathbf{a}_{2}-\mathbf{c}_{2}\right)$ segmentations obtained by the empirically fine-tuned version, in the same iteration that the automated version has converged, and $\left(\mathbf{a}_{3}-\mathbf{c}_{3}\right)$ segmentation results of the automated version

whereas the abnormality is classified as (a) well defined/circumscribed and (b) ill defined. In terms of its severity, the abnormality is defined as either benign or malignant. Figures 7, 8, 9, and 10 depict segmentation results obtained by the automated version using the presented framework as well as by the empirical version in the same iteration that the automated version has converged. 


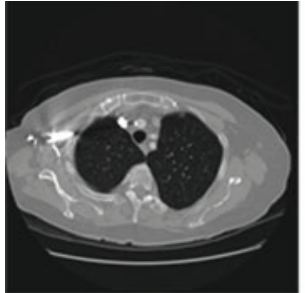

(a)

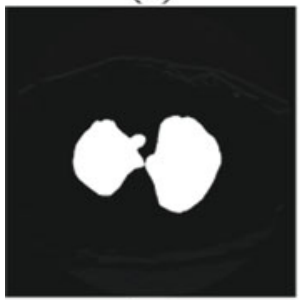

$\left(a_{1}\right)$

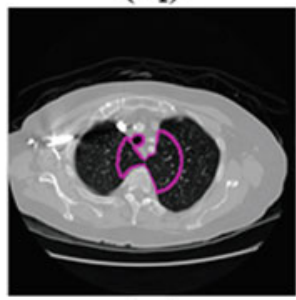

$\left(a_{2}\right)$

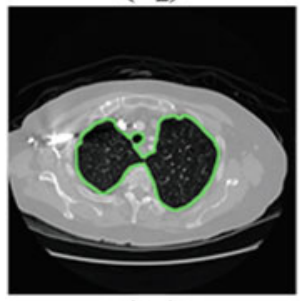

$\left(a_{3}\right)$

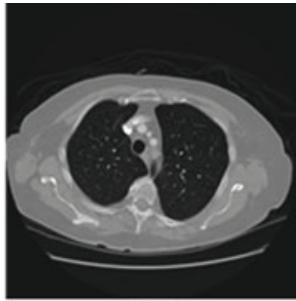

(b)

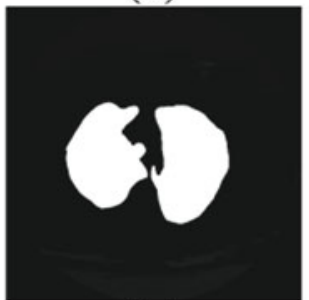

$\left(b_{1}\right)$

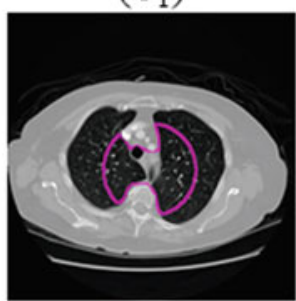

$\left(b_{2}\right)$

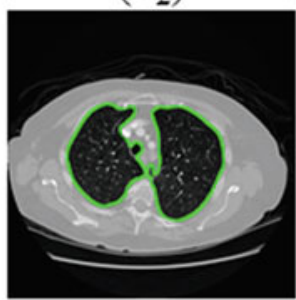

$\left(b_{3}\right)$

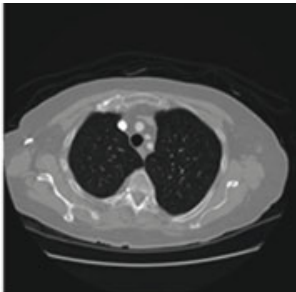

(c)

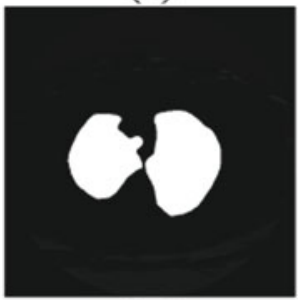

$\left(c_{1}\right)$

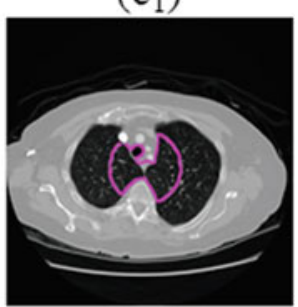

$\left(c_{2}\right)$

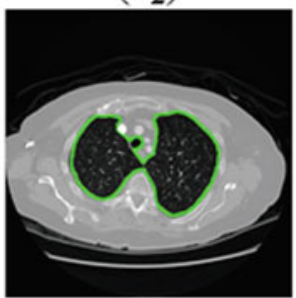

$\left(c_{3}\right)$

Fig. 8 (a-c) Lung CT scans, $\left(\mathbf{a}_{1}-\mathbf{c}_{1}\right)$ ground truth images, $\left(\mathbf{a}_{2}-\mathbf{c}_{2}\right)$ segmentations obtained by the empirically fine-tuned version, in the same iteration that the automated version has converged, and $\left(\mathbf{a}_{3}-\mathbf{c}_{3}\right)$ segmentation results of the automated version

Considering Figs. $7,8,9$, and 10 and by comparing sub-images $\left(\mathrm{a}_{2}\right)-\left(\mathrm{c}_{2}\right)$ to $\left(a_{3}\right)-\left(c_{3}\right)$, it is evident that contour convergence is delayed in the empirically fine-tuned version. However, in the automated version, contour convergence is accelerated since the former is capable of distinguishing randomly oriented, 


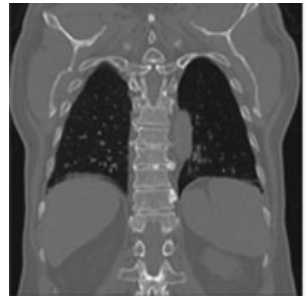

(a)

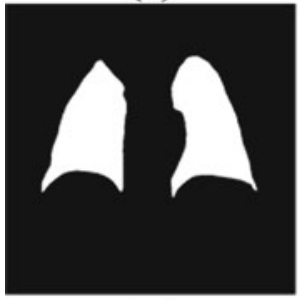

$\left(a_{1}\right)$

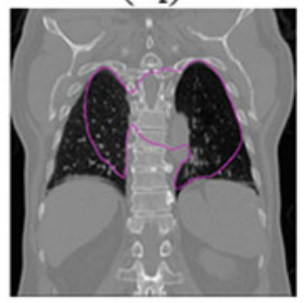

$\left(a_{2}\right)$

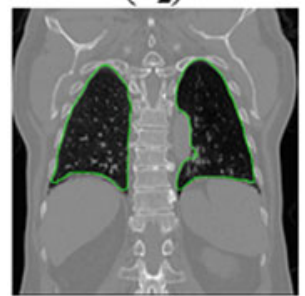

$\left(a_{3}\right)$

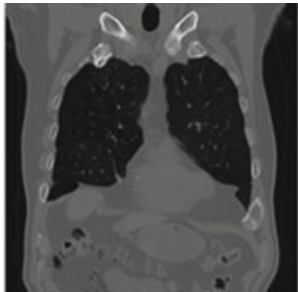

(b)

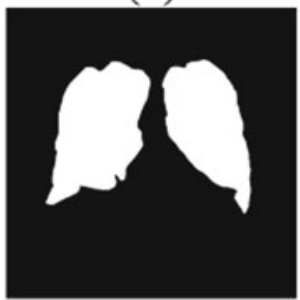

$\left(b_{1}\right)$

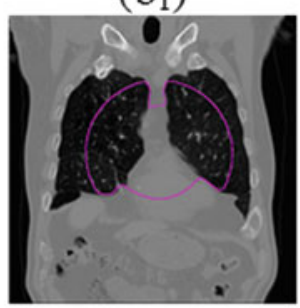

$\left(b_{2}\right)$

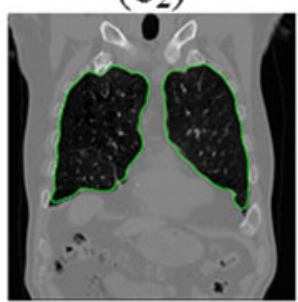

$\left(b_{3}\right)$

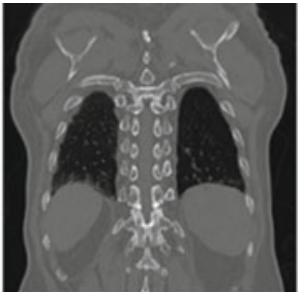

(c)

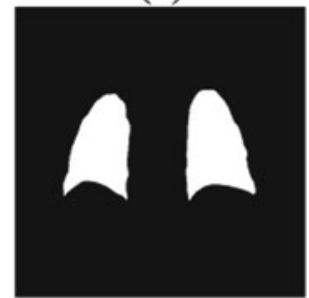

(c $\left.c_{1}\right)$

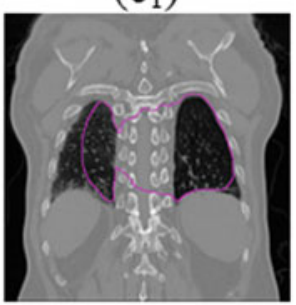

$\left(c_{2}\right)$

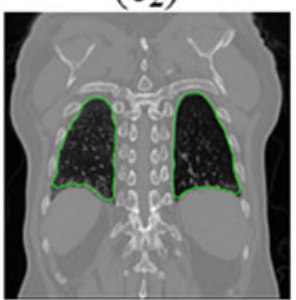

$\left(c_{3}\right)$

Fig. 9 (a-c) Thorax CT scans, $\left(\mathbf{a}_{1}-\mathbf{c}_{1}\right)$ ground truth images, $\left(\mathbf{a}_{2}-\mathbf{c}_{2}\right)$ segmentations obtained by the empirically fine-tuned version, in the same iteration that the automated version has converged, and $\left(\mathbf{a}_{3}-\mathbf{c}_{3}\right)$ segmentation results of the automated version

high-entropy edges from target ones, as explained in Fig. 6. Region-based forces which guide contour evolution are appropriately amplified in unstructured, nontarget edge regions driving the contour away. Hence, iterations dedicated to false local minima, associated with such regions, are avoided. 


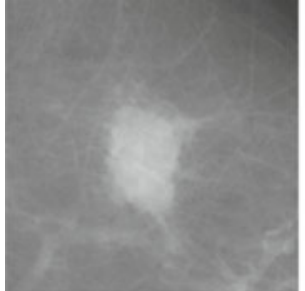

(a)

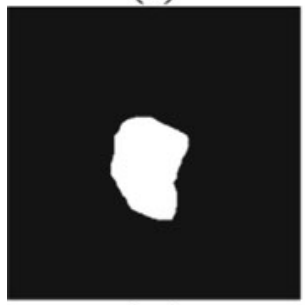

$\left(a_{1}\right)$

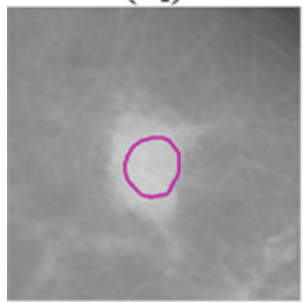

$\left(a_{2}\right)$

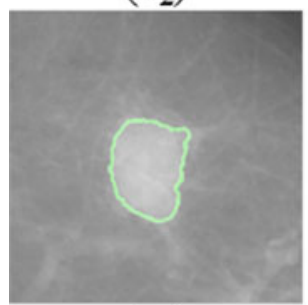

$\left(a_{3}\right)$

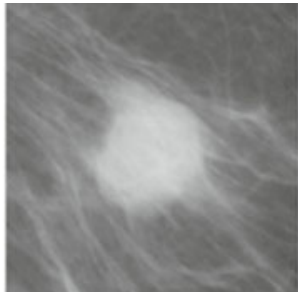

(b)

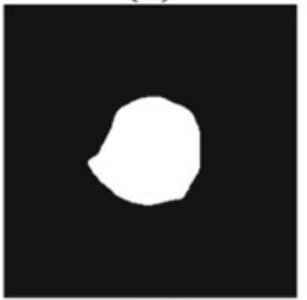

$\left(b_{1}\right)$

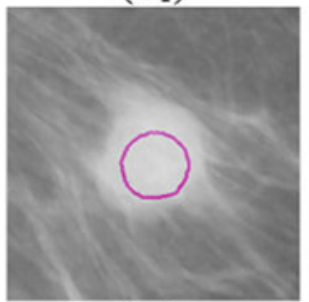

$\left(b_{2}\right)$

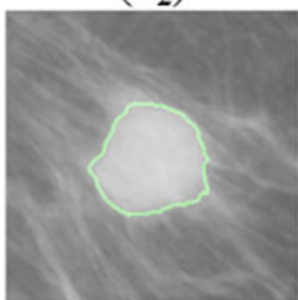

$\left(b_{3}\right)$

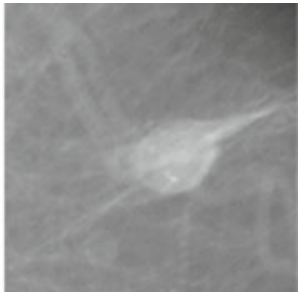

(c)

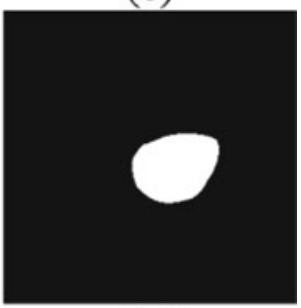

$\left(c_{1}\right)$

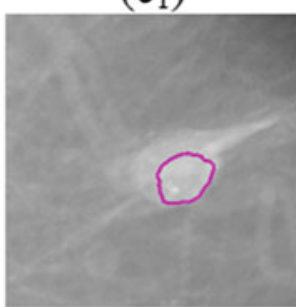

$\left(c_{2}\right)$

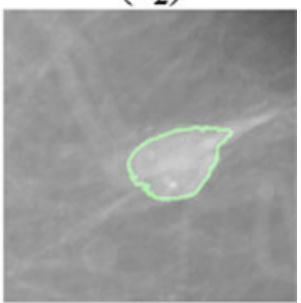

$\left(c_{3}\right)$

Fig. 10 (a-c) Mammographic images containing abnormalities, $\left(\mathbf{a}_{1}-\mathbf{c}_{1}\right)$ ground truth images, $\left(\mathbf{a}_{2}-\mathbf{c}_{2}\right)$ segmentations obtained by the empirically fine-tuned version, in the same iteration that the automated version has converged, and $\left(\mathbf{a}_{3}-\mathbf{c}_{3}\right)$ segmentation results of the automated version

\section{Quantitative Evaluation}

The experimental results are quantitatively evaluated by means of the region overlap measure, known as the tanimoto coefficient (TC) [32], which is defined by:

$$
\mathrm{TC}=\frac{N(A \cap B)}{N(A \cup B)}
$$


Table 1 TC values obtained by the empirical and automated version, in the iteration that the latter has converged

Fig. 11 TC for the segmentations of automated vs. empirical parameterization presented in Table 1

\begin{tabular}{lcll}
\hline & & \multicolumn{2}{l}{ TC $(\%)$} \\
\cline { 3 - 4 } Dataset & Iterations & Empirical & Automated \\
\hline Endoscopy & 6 & $51.4 \pm 3.8$ & $82.3 \pm 1.4$ \\
Lung & 33 & $49.1 \pm 2.2$ & $81.8 \pm 0.3$ \\
Thorax & 23 & $52.5 \pm 4.2$ & $82.7 \pm 0.5$ \\
Mammogram & 19 & $48.3 \pm 6.5$ & $83.2 \pm 1.2$ \\
\hline
\end{tabular}

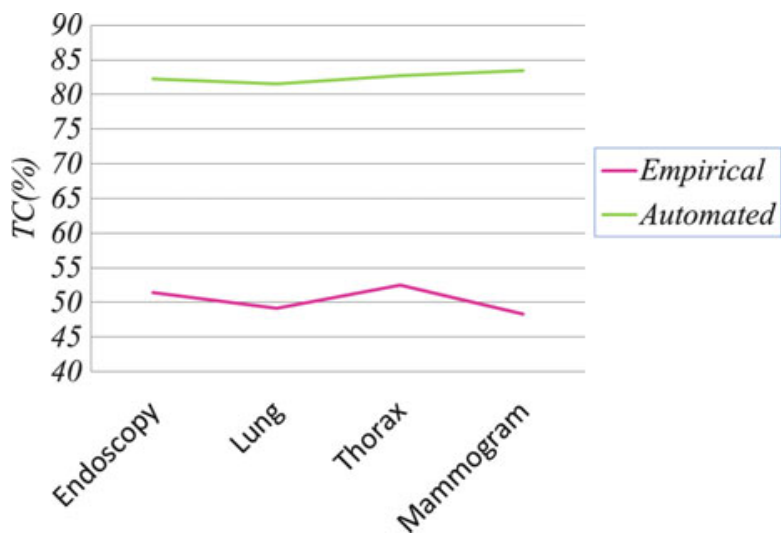

where $A$ is the region identified by the segmentation method under evaluation, $B$ is the ground truth region, and $N()$ indicates the number of pixels of the enclosed region. The automated version achieves an average TC value of $82.9 \pm 1.6 \%$, which is comparable to the TC value of $80.7 \pm 1.8 \%$ obtained by the empirically fine-tuned version, with regards to all images tested. Nevertheless, the automated version converges in 10-20 times less iterations. The empirically fine-tuned version achieves a TC value of $52.4 \pm 11.3 \%$, in the same iteration that the automated version has converged.

Table 1 shows, for each utilized dataset, the iterations that the automated version has converged as well as TC values obtained by the empirical and automated version, for these same iterations. Figure 11 compares the segmentation performance of the automated vs. empirical parameterization for each utilized dataset presented in Table 1.

The experimental results are also evaluated on the convergence rate of both versions by means of the difference mean intensity value (DMI). DMI is calculated between the inside and outside region terms of the contour according to the following algorithm:

\section{$\forall$ Iteration $i$}

1. Calculate inside $\left|I(x, y)-c_{1}\right|^{2}$ and outside $\left|I(x, y)-c_{2}\right|^{2}$ region terms.

2. Normalize and quantize both terms in the range [0,255].

3. Calculate mean values.

4. Calculate DMI. 
Table 2 DMI values obtained by the empirical and automated version, in the early stages of contour evolution

\begin{tabular}{lcc}
\hline & DMI & \\
\cline { 2 - 3 } Dataset & Empirical & Automated \\
\hline Endoscopy & 8.2 & 14.0 \\
Lung & 3.4 & 7.1 \\
Thorax & 5.8 & 8.3 \\
Mammogram & 28.2 & 30.8 \\
\hline
\end{tabular}

During contour evolution, DMI is increased, and once contour converges to the actual target boundaries, DMI obtains its highest value.

Table 2 shows for a sample of each utilized dataset DMI values obtained by the empirical and automated version in the early stages of contour evolution.

It can be observed that DMI reaches higher values in the automated version in the early stages of contour evolution, regardless of the medical imaging modality. This convergence acceleration has been theoretically justified in Section "Parameter-Adjustment Framework."

\section{Conclusion}

Medical image segmentation plays a fundamental role in medical research since it aids MDs' clinical evaluation by providing vital information on abdominal organs. Empirical parameterization in segmentation techniques is not accurate since the segmentation results are dependent on the visual impression of a MD. Thus, it is crucial to develop automated algorithms which are accurate and do not require any user intervention.

In this chapter, a framework for automated adjustment of active contour regularization and data fidelity parameters is presented and applied for medical image segmentation. The presented framework is inspired from the properties of structure tensors. The latter are appropriate descriptors of the orientation coherence of edge regions. This information is accordingly incorporated into the active contour parameters by means of OE. In this light, region-based forces are boosted on nontarget, unstructured regions, driving the contour away and guiding it towards the target, structured ones. Thus, iterations dedicated to false local minima are avoided and contour convergence is accelerated. More importantly, MDs are set free from the laborious process of empirical parameterization, and objectivity is bolstered.

The presented framework is evaluated on abdominal imaging modalities, including colon and lung images as well as on mammographic images, by comparing its segmentation performance with the empirically fine-tuned version. The experimental results demonstrate that the automated version is capable of meliorating contour evolution as well as maintaining a high segmentation quality, comparable to the one obtained empirically. Furthermore, it copes well with the variability of target regions and remains insensitive to noise, artifacts, and heterogeneity. 


\section{References}

1. Shang Y, Yang X, Zhu L, Deklerck R, Nyssen E (2008) Region competition based active contour for medical object extraction. Comput Med Imag Graph 32(2):109-117

2. Wang L, Li C, Sun Q, Xia D, Kao C-Y (2009) Active contours driven by local and global intensity fitting energy with application to brain $\mathrm{mr}$ image segmentation. Comput Med Imag Graph 33(7):520-531

3. Xu J, Monaco JP, Madabhushi A (2010) Markov random field driven region-based active contour model (maracel): application to medical image segmentation. In: Proceedings of the 13th international conference on medical image computing and computer-assisted intervention (MICCAI), vol 13, pp 197-204

4. Ramudu K, Reddy GR, Srinivas A, Krishna TR (2012) Global region based segmentation of satellite and medical imagery with active contours and level set evolution on noisy images. Int J Appl Phys Math 2(6):449-453

5. Loncaric S, Kovacevic D, Sorantin E (2000) Semi-automatic active contour approach to segmentation of computed tomography volumes. SPIE Proc 3979:917-924

6. Jiang H, Cheng Q (2009) Automatic 3D segmentation of ct images based on active contour models. In: Proceedings of the 11th IEEE international conference on computer-aided design and computer graphics, Aug 2009, pp 540-543

7. Plajer IC, Richter D (2010) A new approach to model based active contours in lung tumor segmentation in 3D CT image data. In: Proceedings of the 10th IEEE international conference on information technology and applications in biomedicine (ITAB), Nov 2010, pp 1-4

8. Ma L, Yu J (2010) An unconstrained hybrid active contour model for image segmentation. In: Proceedings of the 10th IEEE international conference on signal processing (ICSP), Oct 2010, pp 1098-1101

9. McIntosh C, Hamarneh G (2007) Is a single energy functional sufficient? Adaptive energy functionals and automatic initialization. In: Proceedings of the 10th international conference on medical image computing and computer-assisted intervention (MICCAI), vol 10, pp 503-510

10. Erdem E, Tari S (2009) Mumford-Shah regularizer with contextual feedback. J Math Imag Vis 33(1):67-84

11. Kokkinos I, Evangelopoulos G, Maragos P (2009) Texture analysis and segmentation using modulation features, generative models and weighted curve evolution. IEEE Trans Pattern Anal Mach Intell 31(1):142-157

12. Pluempitiwiriyawej C, Moura JMF, Wu YJL, Ho C (2005) STACS: new active contour scheme for cardiac MR image segmentation. IEEE Trans Med Imaging 24(5):593-603

13. Tsai A, Yezzi A, Wells W, Tempany C, Tucker D, Fan A, Grimson WE, Willsky A (2003) A shape-based approach to the segmentation of medical imagery using level sets. IEEE Trans Med Imaging 22(2):137-154

14. Keuper M, Schmidt T, Padeken J, Heun P, Palme K, Burkhardt H, Ronneberger O (2010) 3D deformable surfaces with locally self-adjusting parameters-a Robust method to determine cell nucleus shapes. In: Proceedings of the 20th IEEE international conference on pattern recognition (ICPR), Aug 2010, pp 2254-2257

15. Liu X, Cheung YM, Li M, Liu H (2010) A lip extraction method using localized active contour model with automatic parameter selection. In: Proceedings of the 20th IEEE international conference on pattern recognition (ICPR), Aug 2010, pp 4332-4335

16. Iakovidis D, Savelonas M, Karkanis S, Maroulis D (2007) A genetically optimized level set approach to segmentation of thyroid ultrasound images. Appl Intell 27(3):193-203

17. Hsu CY, Liu CY, Chen CM (2008) Automatic segmentation of liver PET images. Comput Med Imag Graph 32(7):601-610

18. Allili M, Ziou D (2008) An approach for dynamic combination of region and boundary information in segmentation. In: Proceedings of the 19th IEEE international conference on pattern recognition (ICPR), pp 1-4 
19. Yushkevich PA, Piven J, Cody H, Ho S, Gee JC, Gerig G (2005) User-guided level set segmentation of anatomical structures with ITK-SNAP. Insight journal special issue on ISC/NA-MIC/MICCAI workshop on open-source software

20. Dong B, Chien A, Mao Y, Ye J, Osher S (2008) Level set based surface capturing in 3D medical images. In: Proceedings of the 11th international conference on medical image computing and computer-assisted intervention (MICCAI), vol 11, pp 162-169

21. Bigun J, Granlund G, Wiklund J (1991) Multidimensional orientation estimation with applications to texture analysis and optical flow. IEEE Trans Pattern Anal Mach Intell 13 (8):775-790

22. Tschumperlé D, Deriche R (2005) Vector-valued image regularization with PDEs: a common framework for different applications. IEEE Trans Pattern Anal Mach Intell 27(4):506-517

23. Khne G, Weickert J, Schuster O, Richter S (2001) A tensor-driven active contour model for moving object segmentation. In: IEEE international conference on image processing (ICIP), Oct 2001, vol 2, pp 73-76

24. Weickert J, Scharr H (2002) A scheme for coherence-enhancing diffusion filtering with optimized rotation invariance. J Vis Comm Image Represent 13(1-2):103-118

25. Larrey-Ruiz J, Verdú-Monedero R, Morales-Sánchez J, Angulo J (2011) Frequency domain regularization of d-dimensional structure tensor-based directional fields. J Image Vis Comput 29(9):620-630

26. Do MN, Vetterli M (2005) The contourlet transform: an efficient directional multiresolution image representation. IEEE Trans Image Process 14(12):2091-2106

27. Katsigiannis S, Keramidas E, Maroulis D (2010) A contourlet transform feature extraction scheme for ultrasound thyroid texture classification. Engineering Intelligent Systems, Special issue: Artificial Intelligence Applications and Innovations, vol 18(3/4)

28. Vetterli M (1984) Multidimensional subband coding: some theory and algorithms. IEEE Trans Signal Process 6(2): $97-112$

29. Chan TF, Vese LA (2001) Active contours without edges. IEEE Trans Image Process 10 (2):266-277

30. http://www.cancerimagingarchive.net

31. Suckling J, Parker J, Dance D, Astley S, Hutt I, Boggis C, Ricketts I, Stamatakis E, Cerneaz N, Kok S, Taylor P, Betal D, Savage J (1994) The mammographic images analysis society digital mammogram database. Experta Medica International Congress Series 1069:375-378

32. Crum WR, Camara O, Hill DLG (2006) Generalized overlap measures for evaluation and validation in medical image analysis. IEEE Trans Med Imaging 25(11):1451-1461 


\section{Biography}

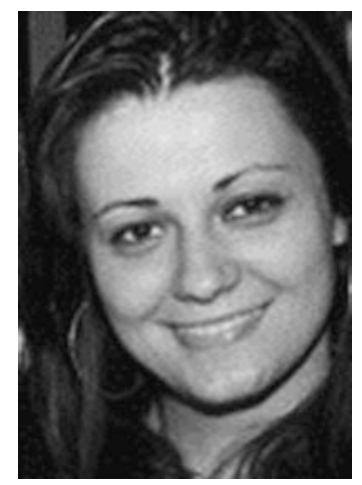

Eleftheria A. Mylona received the B.Sc. degree in Applied Mathematical and Physical Sciences in 2006 from the National Technical University of Athens, Greece, and the M.Sc. degree in Physics in 2007 from the University of Edinburgh, UK. She is currently working towards the Ph.D. degree in Image Analysis at the University of Athens, Greece. For her Ph.D. research, she received a scholarship co-financed by the European Union and Greek National Funds. She has coauthored 11 research articles on biomedical image analysis. Her research interests include image analysis, segmentation, and biomedical applications.

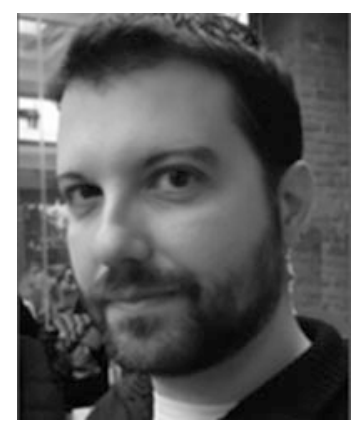

Michalis A. Savelonas received the B.Sc. degree in Physics in 1998, the M.Sc. degree in Cybernetics in 2001 with honors, and the Ph.D. degree in the area of Image Analysis in 2008 with honors, all from the University of Athens, Greece. For his Ph.D. research, he received a scholarship by the Greek General Secretariat for Research and Technology $(25 \%)$ and the European Social Fund $(75 \%)$. He is currently a research fellow in the Dept. of Electrical and Computer Engineering in the Democritus University of Thrace, as well as in ATHENA Research and Innovation Center, branch of Xanthi. In addition, he is a regular research associate of the Dept. of Informatics and Telecommunications of the University of Athens, Greece, as well as of the Center for Technological Research of Central Greece. In the past, he has served in various 
academic positions in the University of Athens, University of Houston, TX, USA, Hellenic Air Force Academy and Technological Educational Institute of Lamia. In 2002-2004 he had been working in software industry as responsible for defining, designing, coding, debugging, testing, integrating, and documenting software modules for real-time systems within the context of various projects. Dr. Savelonas has coauthored more than 40 research articles in peer-reviewed international journals, conferences, and book chapters, whereas he has been actively involved in several EU, US, and Greek R\&D projects. He is a reviewer in prestigious international journals including Pattern Recognition and IEEE Transactions on Image Processing. His research interests include image analysis, segmentation, pattern recognition, 3D retrieval, and biomedical applications.

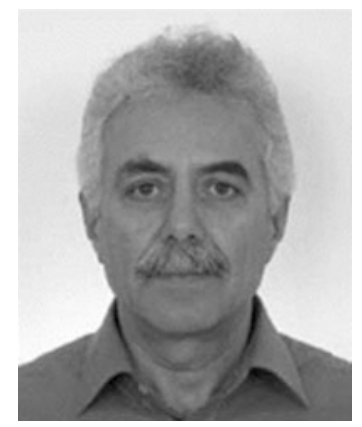

Dimitris Maroulis received the B.Sc. degree in Physics, the M.Sc. degree in Radioelectricity and in Cybernetics with honors, and the Ph.D. degree in Computer Science with honors, all from the University of Athens, Greece. He served as a Research Fellow for three years at the Space Research Department (DESPA) of Meudon Observatory, Paris, France, and afterward he collaborated for more than ten years with the same department. He has also served in various academic positions in the departments of Physics and Informatics of the University of Athens. Currently, he is a Professor in the Department of Informatics and Telecommunications of the same university and leader of the Real-Time Systems and Image Analysis Lab. He has more than 20 years of experience in the areas of data acquisition and real-time systems and more than 15 years of experience in the area of image/signal analysis and processing. He has also been collaborating with many Greek and European hospitals and health centers for more than 15 years in the field of biomedical informatics. He has been actively involved in more than 15 European and National R\&D projects and has been the project leader of 5 of them, all in the areas of image/signal analysis and real-time systems. He has published more than 150 research papers and book chapters, and there are currently more than 1,000 citations that refer to his published work. His research interests include data acquisition and real-time systems, pattern recognition, and image/ signal processing and analysis, with applications on biomedical systems and bioinformatics. 